\title{
Application of numerical model of the photovoltaic module to the practical comparative analysis
}

\author{
Damian Krzesimowski ${ }^{1, *}$, and Artur Pawelec ${ }^{2}$ \\ ${ }^{1}$ Kielce University of Technology, Faculty of Management and Computer Modelling, al. 1000-lecia PP 7, Kielce, Poland \\ ${ }^{2}$ Kielce University of Technology, Faculty of Environmental, Geomatics and Energy Engineering, al. 1000-lecia PP 7, \\ Kielce, Poland
}

\begin{abstract}
This paper includes a model of photovoltaic module based on a basic catalogue data, taking into account the analysis of series and shunt resistance. These resistances are searched with accuracy 0.001 Ohms, but their influence is taken into account in the electrical parameters for each iteration. In addition, the model takes into account changes in environmental parameters: solar radiation intensity and the operating temperature, for which is sought the optimal operating point of the module. This allows to perform a step towards practical applications of the model, including comparative analysis. In the literature numerical models are used to verify the functioning of the PV modules as a function of voltage or current. In the paper is included description of searching the voltage at which the operating point will achieve maximum power for given environmental parameters. This approach to the issue allows to determine the power plane, on which the inverter should move, and this was the first aim of the work. Moreover, it is therefore possible to compare different PV modules without necessity to build the test stands. The output numerical data for different PV modules can be compared with each other and the best model can be chosen to fulfil the condition of the maximum power per day for the desired geographical location. The ability to perform this kind of analysis is the second aim of the work.
\end{abstract}

\section{Introduction}

Nowadays designers of photovoltaic installations have a number of modules to choose from many manufacturers. They have to select the module for specific working conditions. They are supported by data sheets, laboratory tests and numerical tests. The data sheets include basic information about the PV module. The results of laboratory tests are detailed and may contain redundant information that is unnecessary from the investor's point of view. In contrast, computer simulations can be tailored to the investor's expectations. The most important thing is to match the module and its number to the conditions in which the installation is to be operated. The engineer can use laboratory data, but these data, though accurate, should be treated as the output parameters of the module under ideal conditions. It is much more convenient and faster to use a computer program, to input parameters of the selected PV module and to check the results for the given environmental conditions. However, these data are burdened with the modelling error of the PV module. For computational reasons computer models are subject to simplification. This results in differences in the results for laboratory and numerical data. If a single module is considered, the differences will be negligible. However, if the results are extrapolated to the entire system, it may be found that the differences go beyond the statistical error. For this reason, it is necessary to develop numerical models that will generate results on the one hand within the tolerance limits, on the other hand, to ensure that these results are sufficiently consistent with laboratory results.

The most commonly used are single diode equivalent and dual diode equivalent models of photovoltaic cells. Single diode models may, but does not have to, take into account the serial and shunt resistances in the equivalent circuit. The number of photovoltaic cells connected in series to obtain a module is one of the input parameters necessary for the calculation. The number of tools used in the calculation is not large. For analysis purposes, the software MATLAB/Simulink is usually used [1-5]. The proposed models of PV modules included in the papers mentioned above can be compared with the use of this particular software environment. On the other hand, the graphical and numerical results presented in the publications allow to verify own solutions, not necessarily realized with the use of the same tool.

The equivalent circuit model of PV cell is needed in order to simulate its real behaviour. Using the physics of p-n junctions, a cell can be modelled as DC current source in parallel with one diode that represent currents escaping due to diffusion and charge recombination mechanisms. The consideration of the recombination loss leads to more precise model known as one-diode

* Corresponding author: d.krzesimowski@tu.kielce.pl 
model shown in Fig. 1, used in the described research. Two resistances $R_{s}$ and $R_{s h}$ are including to the model the contact resistances and the internal PV cell resistance respectively.

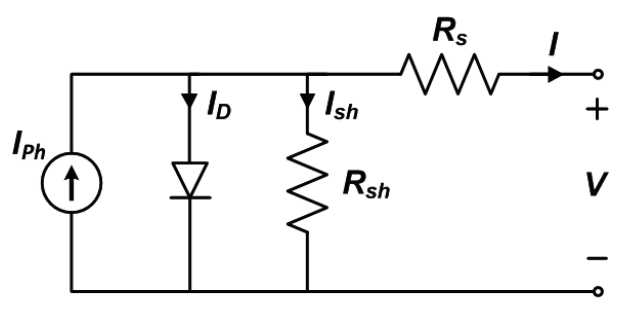

Fig. 1. Single diode equivalent circuit for the silicon photodiode.

The reason for this choice is that this model is well resolved mathematically and sufficiently precise for the purposes of comparative analysis. In addition, only the data sheet of the PV module is required for calculations. However, such sheets do not contain all the necessary data that must be calculated before the final results are obtained. In the study the Scilab software (similar to MATLAB) has been used, and the results are scripts and text files with environmental input data. At the moment no visualization of the proposed mathematical model has been made with the use of the xcos module, but it is possible to present the model as a block diagram or as a description of applied mathematical methods. For the purposes of the paper the last solution had been chosen.

\section{Materials and methods}

The numerical model proposed in this article is based on the following parameters of the PV module:

- typical peak power $P_{m p}$,

- number of cells $N_{s}$,

- short-circuit current $I_{s c}$,

- open-circuit voltage $V_{o c}$,

- current at peak power $I_{m p}$,

- voltage at peak power $V_{m p}$,

- temperature coefficient of short-circuit current $K_{I}$,

- temperature coefficient of short-circuit voltage $K_{V}$,

- ideality (material) factor $A$,

- series resistance $R_{s}$,

- shunt resistance $R_{s h}$.

The last three parameters are not usually listed in data sheets. The ideality factor is defined by the production technology of the PV cell. For the purposes of calculations, the values contained in the table I of the paper [6] has been used. However the values, which in this case are arbitrarily imposed, are a kind of simplification. Semiconductor manufacturing processes may vary from factory to factory, so the exact value of the ideality factor is never known.

The resistances can be accurately determined, it is sufficient to find a series resistance to the purposes of calculation the shunt resistance. In the presented model they are determined with accuracy of $0.001 \mathrm{Ohm}$.
As the environmental conditions STC (Standard Test Condition) values had been used. Additional input data are:

- temperature of the PV module $T_{c}$,

- irradiance $G$,

- Boltzmann constant $k$,

- elementary charge $q$.

The first step towards described model is to determine the modified ideality factor, also called thermal voltage. It can be calculated by using formula (1) based on [4].

$$
a=\left(N_{s} \cdot A k \cdot T_{c}\right) / q
$$

Next step is to determine the reverse saturation current under STC conditions. According to [7] the reverse saturation current of diode at the reference temperature is given by formula (2) with the diode ideality factor added.

$$
I_{0}=I_{s c} /\left\{\exp \left[\left(q \cdot V_{o c}\right) /\left(A \cdot k \cdot T_{c}\right)\right]-1\right\}
$$

The reverse saturation current is temperature dependant but for the purposes of finding series and shunt resistance this fact is not important.

The values of series resistance in an ideal situation tend to zero, while the value of shunt resistance tend to infinity. In fact, the serial resistance is in the range of 0 to $1 \mathrm{Ohm}$ and the shunt resistance is several hundred Ohm. It has already been mentioned that one of the input parameters for determining the shunt resistance is the serial resistance. In the presented model a table of values of serial resistance is created from 0.001 to $1.000 \mathrm{Ohm}$ with an accuracy of $0.001 \mathrm{Ohm}$, therefore consists of 1000 values. Each of these values is substituted with formula (4), according to [4], to determine the corresponding shunt resistance.

$$
A u x E q 3=V_{m p}+I_{m p} \cdot R_{s}-V_{o c}
$$

$R_{s h}=\left(V_{m p}+I_{m p} \cdot R_{s}\right) /\left\{I_{s c}-I_{s c} \cdot[\exp (A u x E q 3 / a)]+I_{s c}\right.$

$\left.\left[\exp \left(-V_{o c} / a\right)\right]-\left(P_{m p} / V_{m p}\right)\right\}$

The iteration starts at $R_{s}=0$ which must increase in order to move the modelled Maximum Power Point until it matches with the $P_{m p}$. There is only one pair of resistances that satisfies this condition. To numerically determine this power output current $I$ should be calculated. One of the essential parameters at this stage is the photocurrent $I_{p v}$ for STC conditions at the currently tested pair of $R_{s}$ and $R_{s h}$. In many papers the calculation is simplified by assuming $I_{p v}=I_{s c}$. Presented model is improved by taking advantage of the iterative solution of $R_{s}$ and $R_{s h}$. Each iteration updates $R_{s}$ and $R_{s h}$ toward the best model solution, so according to [4] formula (5) is introduced in the model.

$$
I_{p v S T C}=I_{s c} \cdot\left[\left(R_{s}+R_{s h}\right) / R_{s h}\right]
$$

Formula (5) uses the resistances $R_{s}$ and $R_{s h}$ to determine $I_{p v} \neq I_{s c}$. The values of resistances are initially unknown but as the solution of the algorithm is refined along successive iterations the values 
of resistances tend to the best solution and (5) becomes valid and effectively determines the light-generated current taking into account the influence of the series and shunt resistances of the array.

Only at this point can be determined the output current and output power. However, it is necessary to prepare an iterative table containing triggering voltage values $V$, in fact generated by the inverter. In the presented model the voltage is determined from 0 to $V_{o c}$ in iteration level calculated by using formula (6).

$$
\text { VstepR }=\left(V_{o c}+0.1 \cdot V_{o c}\right) / 100
$$

The margin $0.1 \cdot V_{o c}$ is intended to improve readability of charts. For the purpose of determining the resulting current, formula (8) had been used according to [1].

$$
\begin{gathered}
A u x E q 7=V+I R_{s} \\
I=I_{p v}-I_{0} \cdot[\exp (A u x E q 7 / a)-1]-A u x E q 7 / R_{s h}
\end{gathered}
$$

Iterative numerical solution of formula (8) is impossible. In the presented model a patch has been introduced that enables the generation of resistance pairs and the corresponding calculated maximum power, bypassing the problem of the presence of the current $I$ on both sides of the formula (8). It consists of three elements: the first is the accuracy of the determination of the serial resistance. The second is the number of iterations for determining the power characteristics. The third and most important is the determination of the current $I$ with using the formula (8) based on the value of this current determined in the previous iteration. Therefore the formula (7) has been redefined in (9).

$$
\operatorname{AuxEq7}(i)=V(i)+I(i-1) \cdot R_{s}
$$

In order to minimize the error thus introduced into the final result, an experimentally determined value of 1.0004 was applied in the formula which describes the power for the current iteration.

$$
P_{m p, e}(i)=V(i) \cdot I(i) \cdot 1.0004
$$

The final result is the calculated power graph plotted on the $P_{m p}$ power diagram from the data sheet and the pair table $R_{s}, R_{s h}$ and $P_{m p, e}$. The solution is the pair of resistance for which $P_{m p, e} \cdot P_{m p}$. The pairs for which $R_{s h}<0$ or $P_{m p, e}<0$ should be rejected.

It is worth noting that the parameter $A$ is taken onto account in calculations, and is the only arbitrarily determined parameter. Because it cannot be sure whether the selected value is correct, the obtained resistance values cannot be treated as true. This means that in the calculations not only pair of $R_{s}$ and $R_{s h}$ is sought but the combination of three values: $A, R_{s}$ and $R_{s h}$. If for example instead of $A=1.2$ it is assumed to be $A=1.3$ then the values of resistance pairs will be different.

Based on the conclusions of one of the paper on this issue [8] it can be assumed that a change in the ideality factor entails a change in the resistances, that no significant differences in the power characteristics of a specific PV module can be found. The condition is the consistent use in the calculations predetermined value $A$ and calculated on the basis of values $R_{s}$ and $R_{s h}$. For the purpose of coarse error determination in the final results, a value for the test PV module had been assumed as $A=1.2$ and $A=1.3$, then the characteristics of $I(V), P(V)$ and $F F(V)$ has been obtained. The Fill Factor $(F F)$ indicator had been introduced to the calculations for the purposes of evaluating the performance of the test module, based on the solution presented in [9]. This indicator is described by the formula (11)

$$
F F(i)=P_{m}(i) /\left(V_{o c} \cdot I_{s c}\right)
$$

The results of searching the resistances are presented in Fig. 2. For the purposes of this paper to the calculation module Bruk-Bet Solar BEP300 had been used with catalogue parameters listen in Table 1.

Table 1. Catalogue parameters of the tested PV module.

\begin{tabular}{|c|c|}
\hline type & poly-Si \\
\hline $\mathrm{P}_{\mathrm{mp}}$ & $300 \mathrm{~W}$ \\
\hline $\mathrm{N}_{\mathrm{s}}$ & 72 \\
\hline $\mathrm{I}_{\mathrm{sc}}$ & $8.95 \mathrm{~A}$ \\
\hline $\mathrm{V}_{\mathrm{oc}}$ & $44.2 \mathrm{~V}$ \\
\hline $\mathrm{I}_{\mathrm{mp}}$ & $8.4 \mathrm{~A}$ \\
\hline $\mathrm{V}_{\mathrm{mp}}$ & $35.8 \mathrm{~V}$ \\
\hline $\mathrm{K}_{\mathrm{I}}$ & $0.049 \% /{ }^{\circ} \mathrm{C}$ \\
\hline $\mathrm{K}_{\mathrm{V}}$ & $-0.32 \% /{ }^{\circ} \mathrm{C}$ \\
\hline
\end{tabular}

On the assumption $A=1.2$ value $R_{s}=0.267 \mathrm{Ohm}$, $R_{s h}=900.124 \mathrm{Ohm}$, but if $A=1.3$ then the result of calculations is: $R_{s}=0.22 \mathrm{Ohm}, R_{s h}=3143.122 \mathrm{Ohm}$. The next step was to compare $I(V)$ and $P(V)$ characteristics and value of $F F$ for the STC conditions. Results are presented in Fig. 3 and Fig. 4. For ideality factor equal to 1.2 the FF point is obtained for $V=36.66$ $V$ and for ideality factor equal to 1.3 the FF point is obtained for $V=36,71 \mathrm{~V}$. The difference is only $0.05 \mathrm{~V}$ and the absolute difference is equal to $0.14 \%$. The same can be claimed about the value of $F F$, the values for $A=$ 1.2 and $A=1.3$ are almost the same. The result of finding a FF point is presented in Fig. 5. There is an insignificant change in shape of the characteristics marked in red and blue, but the most important point on the graph is always in the same place. 


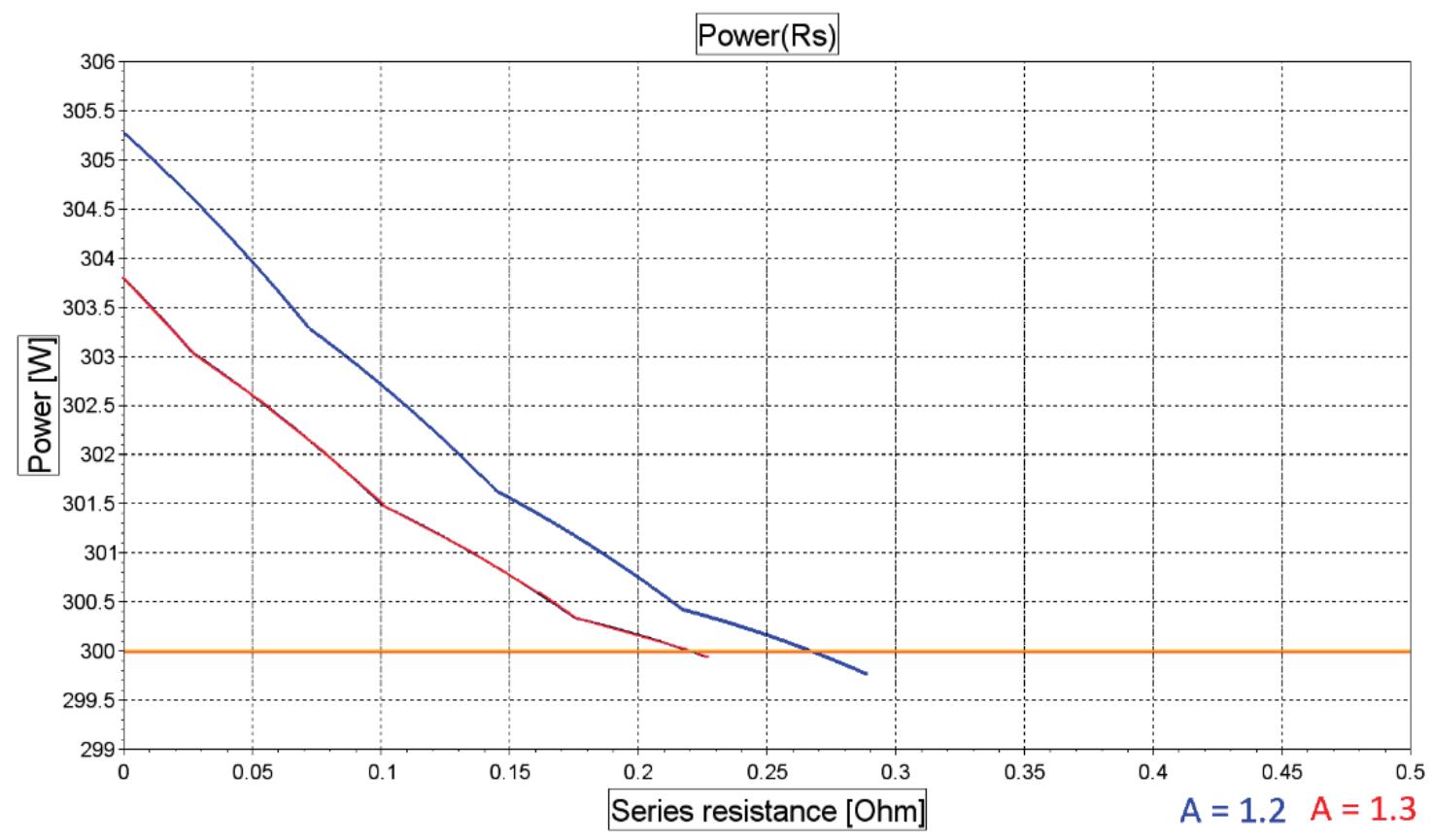

Fig. 2. Results of searching the series resistance for ideality factor (A) equal to 1.2 and 1.3.

\section{Results and discussion}

To verify the numerical model the power characteristics of a specific PV module should be experimentally determined over given interval of time and then the results should be compared with the obtained by using mathematical method. There are few such verifications in literature, the presented model allows it. The ability to use measured environmental parameters and use them to simulate continuous operation of the PV module with known catalogue parameters had been implemented. This is undoubtedly a great advantage of the presented solution, it allows to simulate PV modules without the need to build test stands.

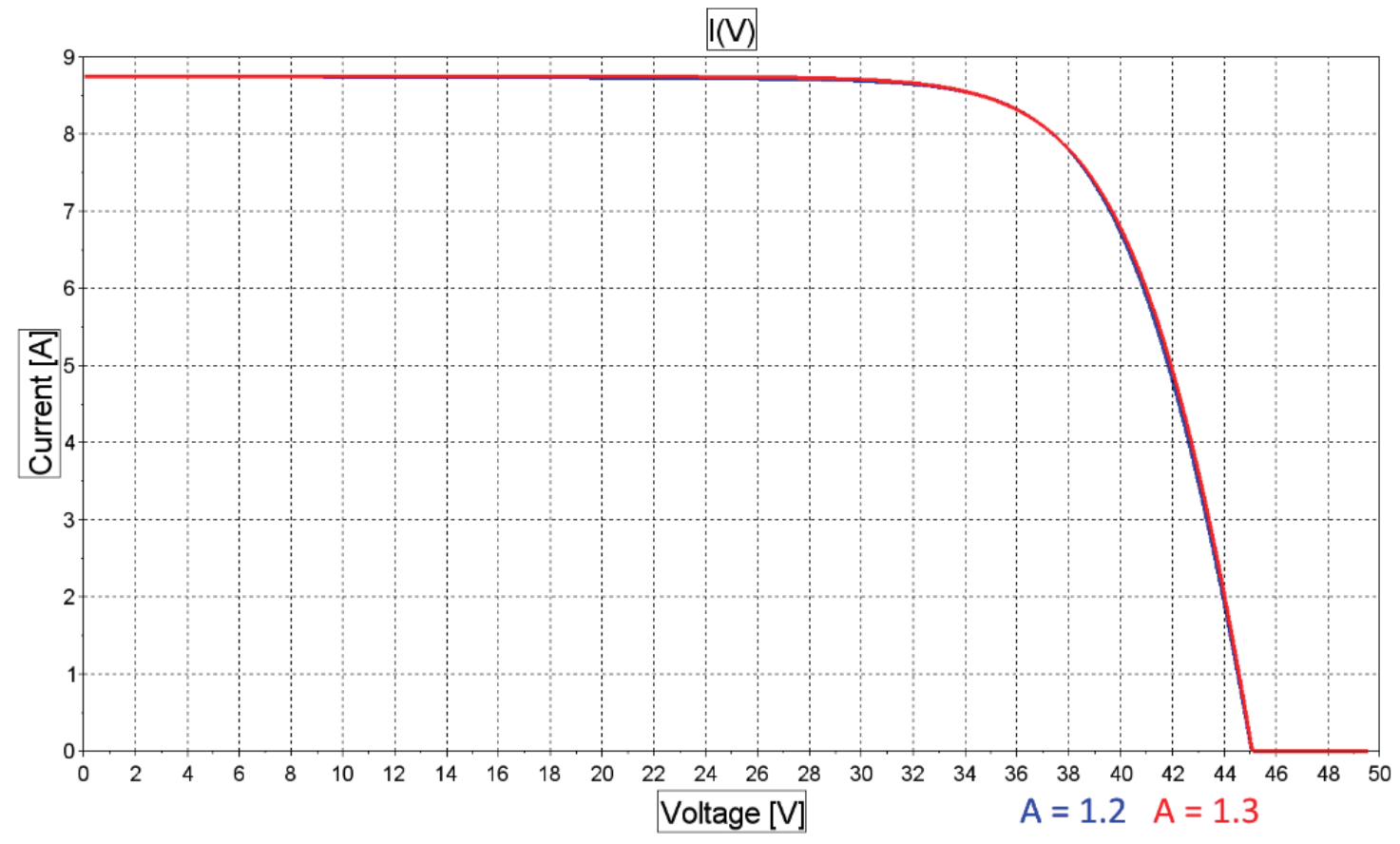

Fig. 3. I(V) characteristics for ideality factor equal to 1.2 and 1.3 under STC conditions. 


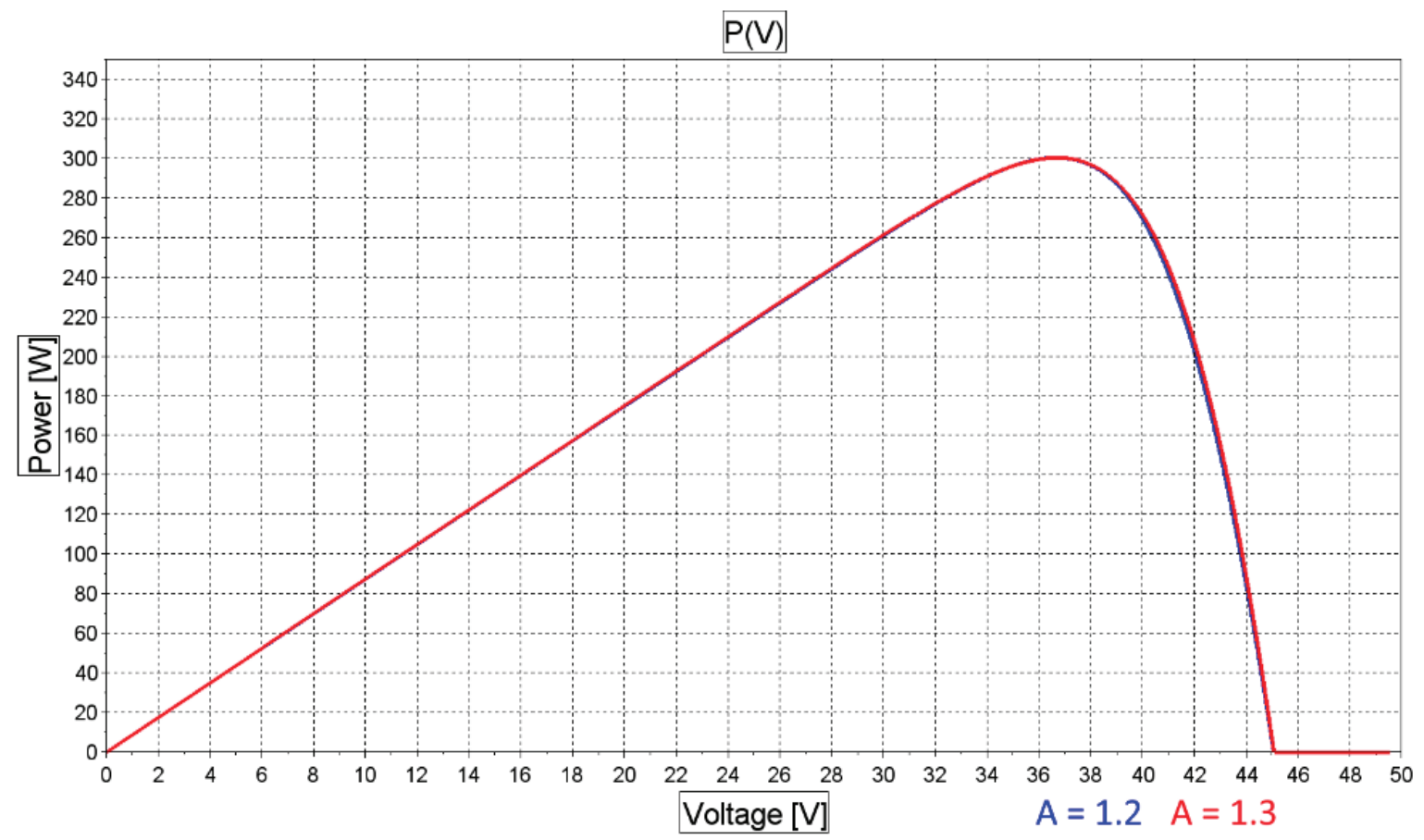

Fig. 4. $\mathrm{P}(\mathrm{V})$ characteristics for ideality factor equal to 1.2 and 1.3 under STC conditions.

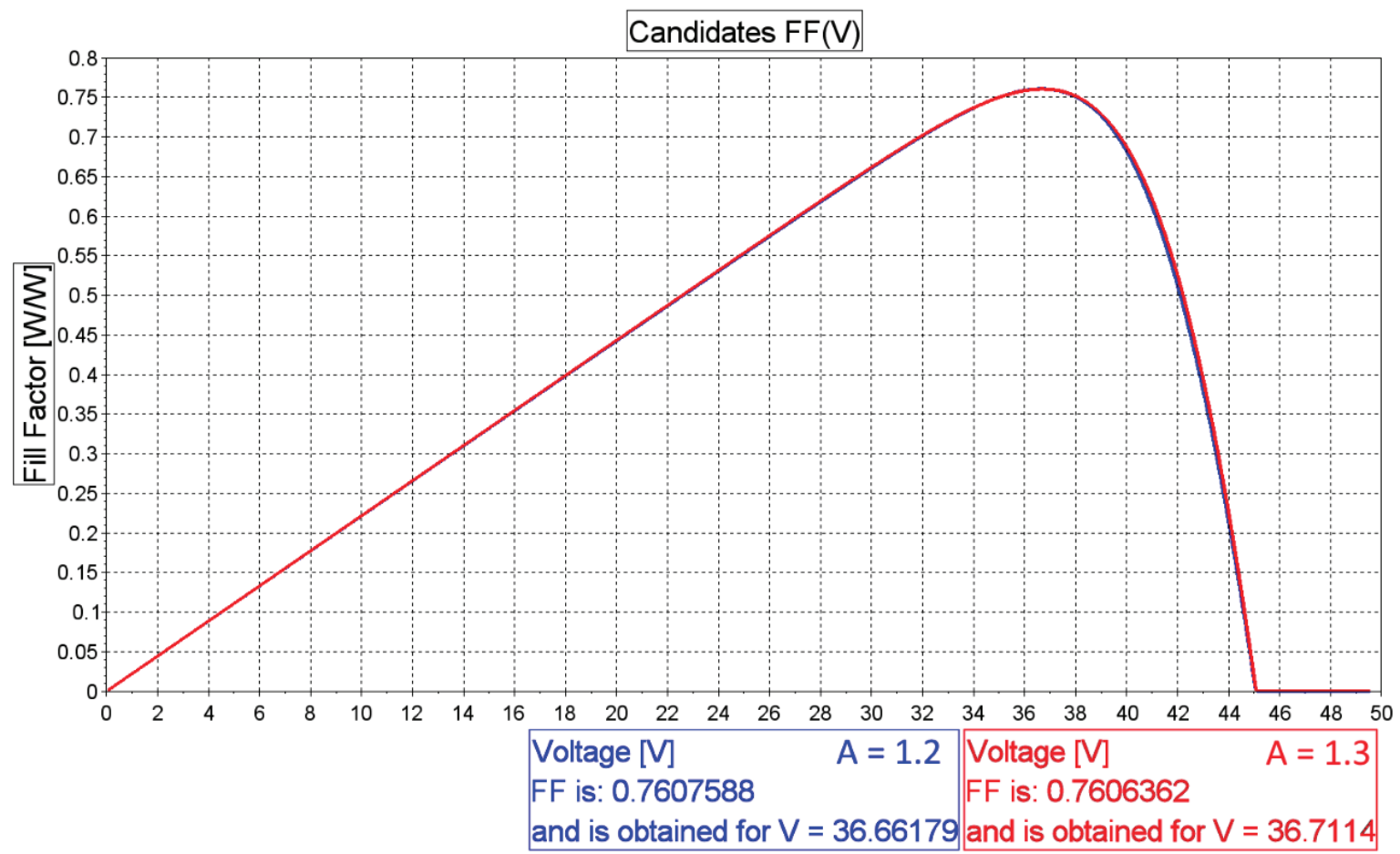

Fig. 5. $\mathrm{FF}(\mathrm{V})$ candidates characteristics for ideality factor equal to 1.2 and 1.3 under STC conditions.

The key to achieving a satisfactory final solution is the self-regulation mechanism of the voltage applied to the module according to the maximum power criterion.

The search of this power is an iteration method and is carried out with every change of work parameters of the module. The iterative control parameter is voltage $V$, calculations start with 0 with the iteration level specified by the formula (12). The iteration loop is performed 1000 times.

$$
\text { VstepP }=\left(V_{o c}+0.1 \cdot V_{o c}\right) / 1000
$$

The modified ideality factor is also calculated 
according to (1). The saturation current is calculated according to [10] with using the formula (14), which for the STC conditions assumes the value of the formula (2).

$$
\begin{gathered}
\Delta T=T_{c}-T_{S T C} \\
I_{0}=\left(I_{s c}+K_{I} \cdot \Delta T\right) /\left\{\exp \left[\left(V_{o c}+K_{V} \cdot \Delta T\right) / a\right]-1\right\}
\end{gathered}
$$

This improvement aims to match the open-circuit voltages of the model with the experimental data for a very large range of temperatures.

Formula (14) is obtained from (2) by including in the equation the current and voltage coefficients $K_{I}$ and $K_{V}$.

The saturation current $I_{0}$ is strongly dependent on the temperature and (14) proposes a different approach to express the dependence of $I_{0}$ on the temperature so that the net effect of the temperature is the linear variation of open-circuit voltage according to the practical voltage/temperature coefficient.

For calculation purposes it is also necessary to determine the photocurrent for the STC conditions with using the formula (5) and the photocurrent taking into account the temperature, which according to [1] can be expressed by the formula (15).

$$
I_{p v}=\left(I_{p v S T C}+K_{I} \Delta T\right) \cdot\left(G / G_{S T C}\right)
$$

At this stage it is possible to determine the output current with the iterative method using formulas (8) and (9). 1000 values of $I(i)$ are obtained, which subsequently multiplied with the appropriate results of $V(i)$ allow to determine the power $P(i)$. Also in this case the problem of numerical determination of the current $I(i)$ had been solved by using values of $I(i-1)$ and the correction of resultant value of power with the formula (10).

It remains to find such a pair $V(i)$ and $I(i)$ for the resulting power to be the greatest. Since the voltage iteration starts at 0 and ends at 1.1 $V_{o c}$ maximum is sought after all possible power characteristics. This is done at the expense of some computing power, which is not a problem as long as changes in the operating parameters of the module occur rarely enough.

The Fill Factor $(F)$ indicator is calculated in each iteration and the maximum value of $F F$ corresponds to the most optimal voltage $V$ that should be applied for the PV module.

For verification of the model and its future commercial uses, the presented solution will generate a power graph over time. Sample graphs from data collected in every 5 minutes are shown in Figs. 6 and 7 . These graphs can be compared with the experimental data and firstly can help to determine the errors of the model and secondly can help to make the necessary corrections in the form of constants used in the formulas.

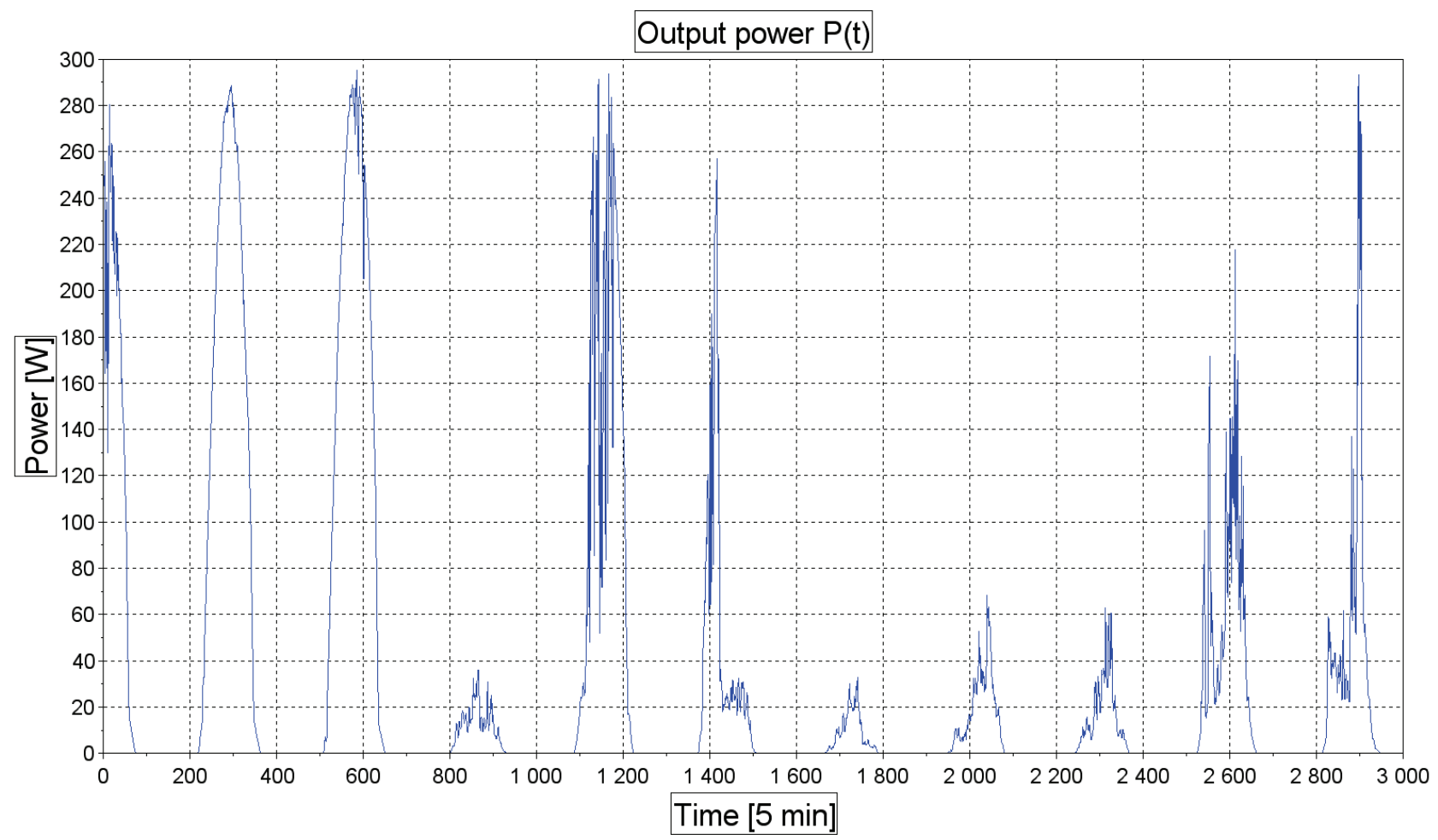

Fig. 6. Characteristics of output power of tested PV module based on the real work conditions. Sampling time is 5 min. 


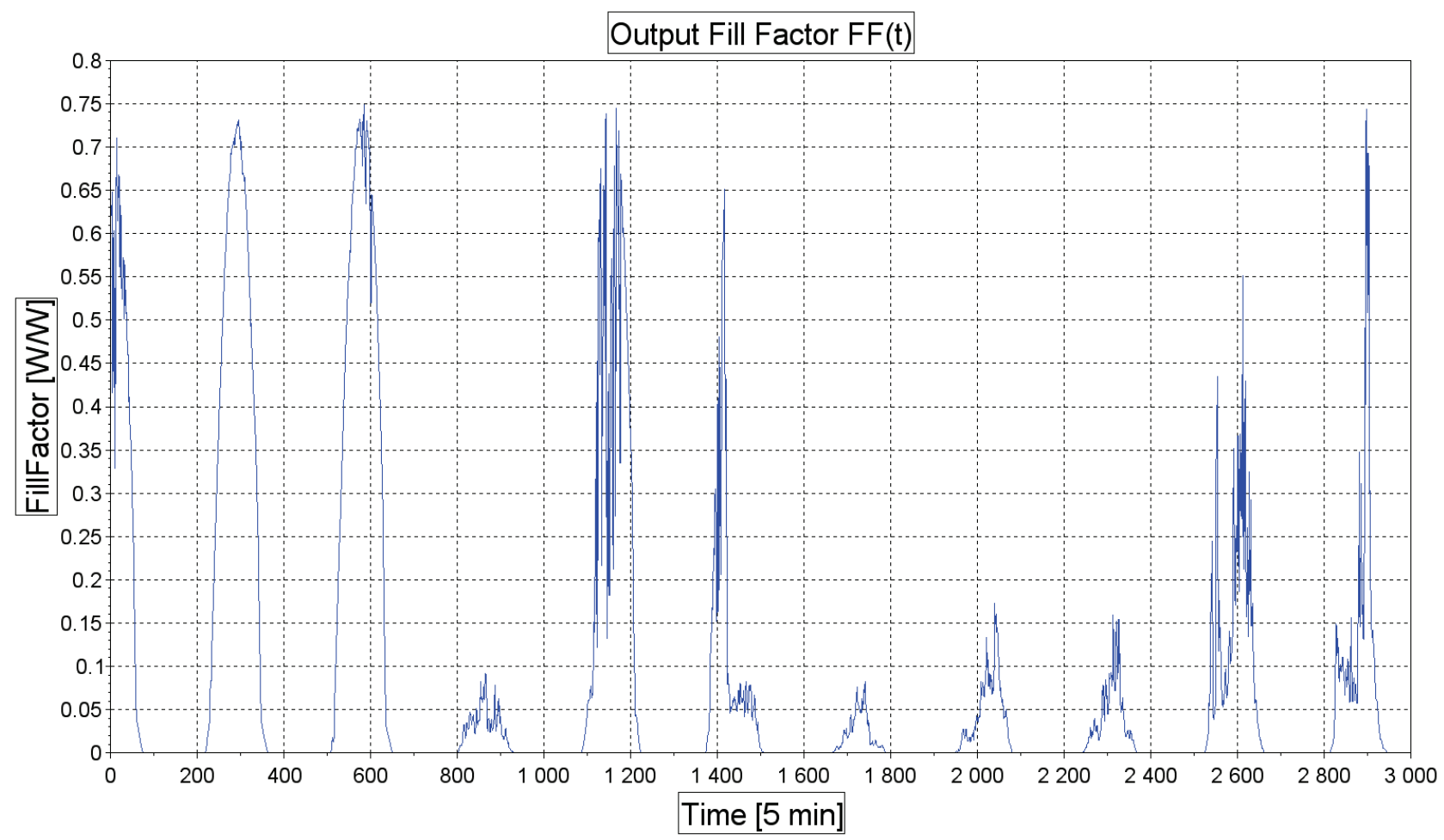

Fig. 7. Characteristics of output Fill Factor of tested PV module based on the real work conditions. Sampling time is 5 min.

\section{Conclusions}

The numerical model of the photovoltaic module presented in this paper is one of the most accurate in the literature. It is based on commonly known mathematical formulas that have been slightly processed for the purpose of increasing the accuracy of the model. The problem of being unable to find a numerical solution of the equation to the output current in a single diode equivalent model with series and shunt resistance had been solved. This had been achieved at the expense of time to obtain solutions and negligible mistake compensated numerically. The result is a new mathematical tool that can be used to compare PV modules with different parameters under the same operating conditions. The novelty is that there is no need to build test stands composed of different PV modules, as well as the ability to get results in minutes, not in a dozen days or so.

The method of comparing the results of undertaken in such way calculations remains to solve. The most important parameter from the investor's point of view is the output power. If modules of different typical peak power are tested, standardisation is required but only after the final results are obtained. In a sense, a parameter that can be helpful is determined from the formula (11) Fill Factor. It allows to determine the performance of a particular module and compare that value with those obtained for other modules without having to use the module's power parameter. Another way to compare modules can be the sum of the output power calculated after the iteration of the operating parameters. In this situation, the investor obtains a graph allowing to evaluate the dynamics of the module's performance under the same conditions. However, this parameter requires mathematical expression, easy to modify and practical implementation. Third way is to use a standardisation of the output power. The output power value for each of the tested PV modules may be reduced to an arbitrarily determined value, e.g. $1 \mathrm{~kW}$. This method would allow to plot the normalised power characteristics of a given module in the irradiance function. This chart could be the most important information for the investor on the data sheet, if it would be placed there.

\section{References}

1. D. Bonkoungou, Z. Koalaga, D. Njomo, Int. J. Emerg. Technol Adv. Eng. 3, 3, 493-502 (2013)

2. J. Park, H-g.K. Yongyun Cho, C. Shin, Adv. Sci. Technol. Lett. 73, 147-155 (2014)

3. B. Alsayid, Int. J. Comput. Technol. Electron. Eng. 1, 3, 6-11 (2012)

4. H. Bellia, R. Youcef, M. Fatima, J. Astron. Geophys. 3, 53-61 (2014)

5. X.H. Nguyen, M.P. Nguyen, Springer Environ. Syst. Res. 4, 24 (2015)

6. H.-L. Tsai, C.-S. Tu, Y.-J. Su, Proceedings of the World Congress on Engineering and Computer Science (2008) 
7. A. El Shahat, J. Power Technol. 94, 1, 50-66 (2014)

8. V.V. Ramana, D. Jena, D.N. Gaonkar, Int. J. Renew. Energy Res. 6, 3, 970-974 (2016)

9. W. Abd El-Basit, A.M., El-Maksood, F., ElMoniem Saad Soliman, Leonardo J. Sci. 23, 13-28 (2013)

10. M.G. Villalva, J.R. Gazoli, E.R. Filho, IEEE Trans. Power Electron. 24, 5, 1198-1207 (2009) 